\title{
RAINBOW HOLOGRAPHIC INTERFEROMETRY
}

\author{
F.T.S. YU ${ }^{1}$ and Hsuan CHEN ${ }^{2}$ \\ Electro-Optics Laboratory, Electrical and Computer Engineering Department, \\ University of Michigan, Ann Arbor, Michigan 48104, USA
}

Received 9 January 1978

A one-step rainbow holographic process is applied to holographic interferometry. Experimental demonstrations of double exposure and time-average interferograms are included. Comparison is made with the conventional holographic process. We found that the rainbow process provides better equality and less speckle noise.

\section{Introduction}

Holographic interferometry is one of the most interesting and important applications of holography. However, in most cases, coherent illumination is required for the reconstruction process. Experience indicates that conventional holographic interferometry has the following shortcomings. First, a coherent source is required for reconstruction and second, significant speckle noise will be present.

We propose a one-step rainbow holographic interferometric process in which these two shortcomings of conventional holographic interferometry may either be removed or improved. As noted, the making of a rainbow hologram requires the recording of a real holographic image of an object through a narrow slit [1-3]. Consequently, if the rainbow hologram is illuminated by a monochromatic light source, a real image is formed, which is limited in its positions of viewability. However, if the rainbow hologram is illuminated by a white light source, the hologram image of the slit will disperse the light into a full view holographic image in full rainbow colors. Thus, the basic reconstruction process of a rainbow hologram is to reproduce a real image of the slit between the hologram image of the object and the observer. We have shown in a previous paper [4] the

${ }^{1}$ On Sabbatical leave from the Department of Electrical and Computer Engineering, Wayne State University, Detroit, Michigan 48202.

2 On Sabbatical leave from the Department of Physics, Saginaw Valley State College, University Center, Michigan 48710.

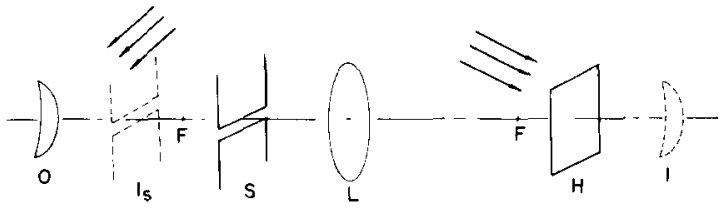

Fig. 1. One-step rainbow holographic process for pseudoscopic imaging. $O$, object; $I$, image of the object; $S$, narrow slit, $I_{S}$, image of the slit; $\mathrm{L}$, imaging lens; $\mathbf{H}$, recording plate; and $\mathbf{F}$, focal point of the lens.

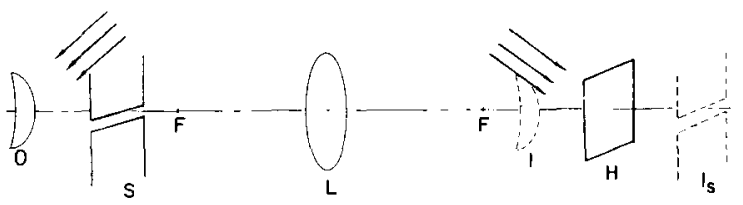

Fig. 2. One-step rainbow holographic process for orthoscopic imaging. $\mathrm{O}$, object; $\mathrm{I}$, image of the object; $\mathrm{S}$, narrow slit; $\mathrm{I}_{\mathbf{S}}$, image of the slit; $L$, imaging lens; $H$, recording plate; and $F$, focus point of the lens.

same goal can be achieved by a one-step rainbow holographic recording, as shown in figs. 1 and 2 . In contrast with the two-step process $[1-3]$, construction of a master hologram is avoided.

\section{Rainbow holographic interferometry}

We note that the optical system for one-step rainbow holographic interferometry is similar to that of conven- 
tional holographic interferometry [5-9], except an imaging lens and a narrow slit are inserted between the object and the recording plate, as shown in fig. 1 . Thus, a virtual image of the slit is formed between the object and the slit, but a real image of the object is formed behind the recording plate. We see that the depth of the real image in its relation to the slit is inverted by the lens. In readout, we require that the slit image be formed downstream from the object image. Therefore, the conjugate image of the hologram must be used, and the image which is viewed by the observer through the slit image is pseudoscopic. We note that by a slight modification of fig. 1, an orthoscopic hologram image can be observed, as shown in fig. 2. We note, however, that in holographic interferometry the required measurements can be carried out equally well whether the image be orthoscopic or pseudoscopic.

A double exposure rainbow holographic interferogram was made using an aluninum strip under stress for an object. A first exposure was made, when the object was under stressed, and a second exposure was made when the mechanical stress was increased slightly. Fig. 3 shows the virtual image, formed by white light illumination. In order to compare this result with that obtained from the conventional technique, the experiment was repeated exactly as before except without the slit. The readout was done with coherent illumination resulting in the image shown in fig. 4. Comparison of figs. 3 and 4 shows the rainbow holographic interferogram to provide higher contrast fringes, and less speckle noise. Also the rainbow image was far brighter even though the illumination $\left(\mathrm{mW} / \mathrm{cm}^{2}\right)$ on the hologram was lower.

In the application to time-average holographic interferometry, a rainbow hologram was made by the same

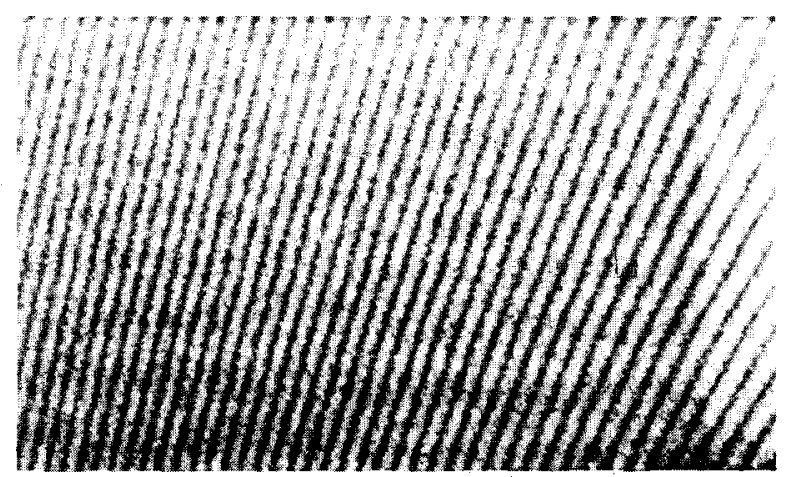

Fig. 3. A double exposure rainbow holographic interferogram.

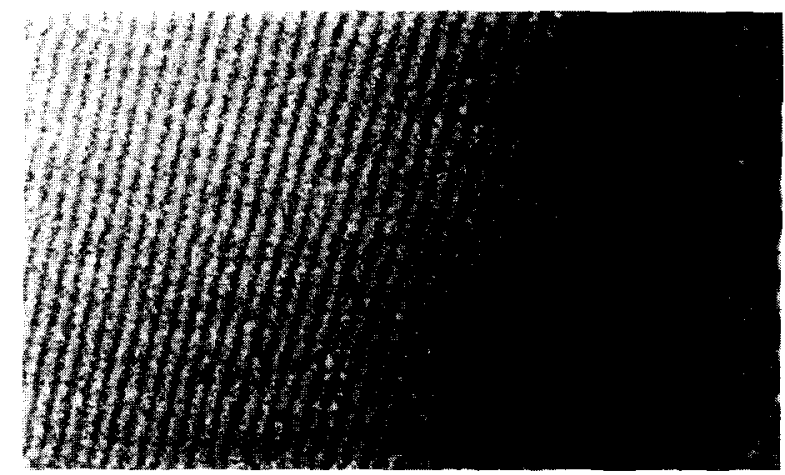

Fig. 4. A conventional double exposure holographic interferogram.

optical setup (fig. 1) using a loudspeaker of about 5 $\mathrm{cm}$ in diameter as a vibrating object. The speaker was driven by a sinusoidal signal of about $1350 \mathrm{~Hz}$. Fig. 5 shows the result of a time-average rainbow holographic interferogram. To compare with the conventional timeaveraging technique, a hologram was made of the same speaker with the same optical setup but without the slit. The image was formed with coherent illumination, as shown in fig. 6 . Comparison of figs. 5 and 6 shows again that the rainbow method results in a higher contrast fringes and less speckle. Also, the image was brighter. Figs. 7 and 8, enlargements of corresponding portions of figs. 5 and 6 , show the severity of speckle to be much greater in the conventional method; in particular the grating structure at the center portion of the speaker is in the conventional case completely

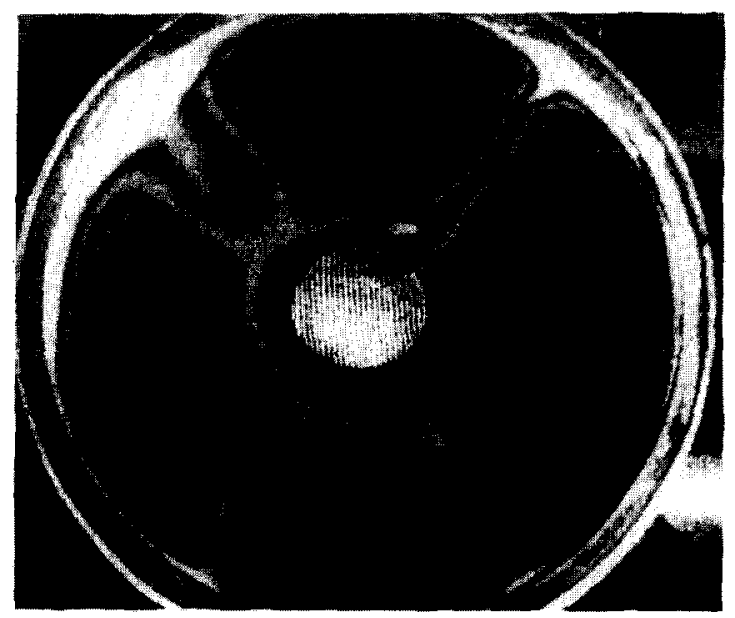

Fig. 5. A time-average rainbow holographic interferogram. 


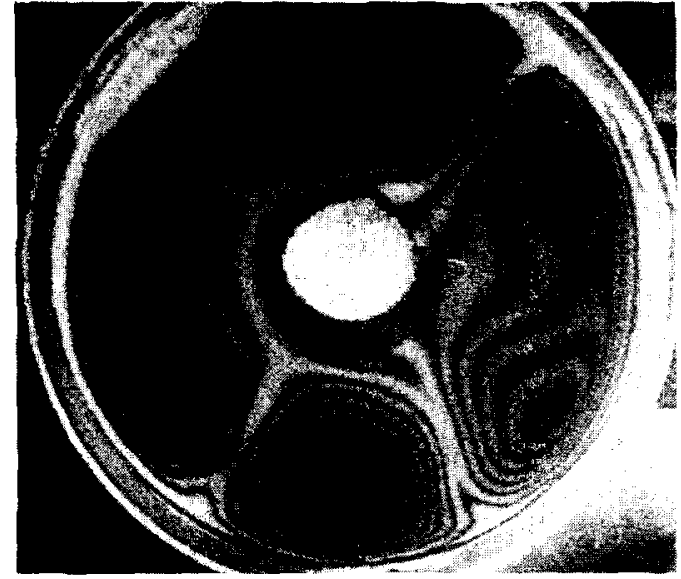

Fig. 6. A conventionai time-average holographic interferogram.

washed out by the speckle noise, but is quite visible in the rainbow case. Thus, we expect a rainbow holographic interferogram to offer somewhat better resolution as compared with a conventional holographic interferogram.

\section{Conclusion}

The one-step rainbow process can be easily applied to most cases of holographic interferometry, yielding the advantages of white light readout, brighter images, less speckle noise, and better resolution. In addition, although not shown here, orthoscopic image interfero-

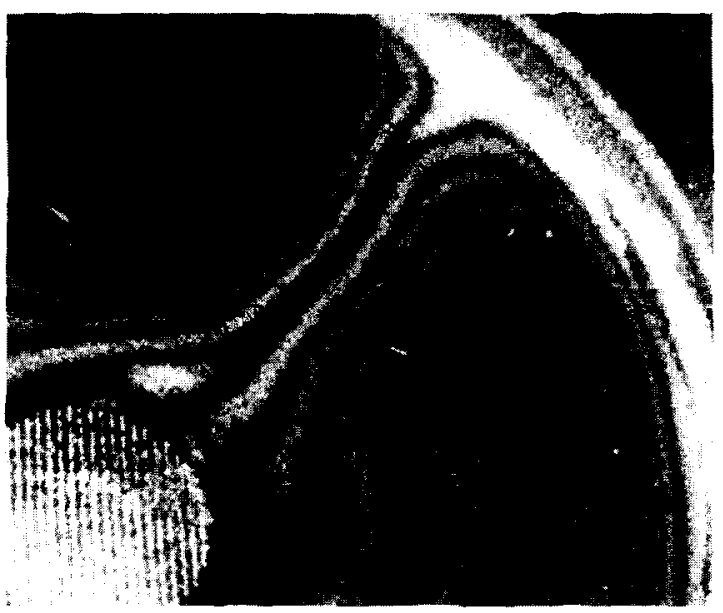

Fig. 7. An enlarged portion of fig. 5 .

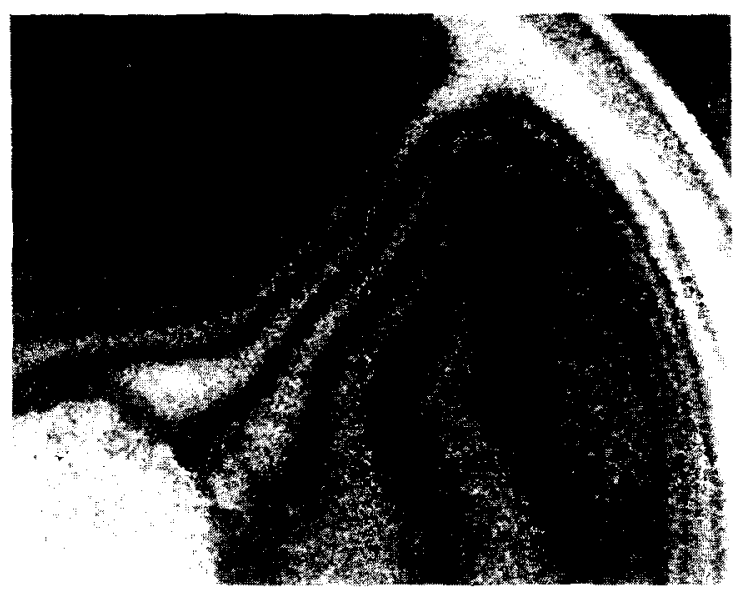

Fig. 8. An enlarged portion of fig. 6 .

grams can also be easily obtained by the optical step up of fig. 2. However, as was noted, pseudoscopic images will normally serve just as well.

We also noted that the two-step rainbow process could have produced the results shown here; however, it is more cumbersome and requires a two-step coherent recording process; therefore, a higher speckle noise in the holographic interferogram is unavoidable.

We wish to acknowledge Professor E.N. Leith for his valuable comments and suggestions during the course of this work, and we acknowledge the support of the National Science Foundation (Grant Eng.7702628.)

\section{References}

[1] S.A. Benton, J. Opt, Soc. Am. 59 (1969) 1545 A.

[2] S.A. Benton, White light transmission/reflection holographic imaging, Proc. ICO Conf. on Application of holography and optical information processing, Jerusalem, Israel, 1976, eds. E. Marom, A.A. Friesem and E. Wiener-Avner, pp. 401 409.

[3] E.N. Leith, Sci. Am. 235 (1976) 80.

[4] H. Chen and I.T.S. Yu, Optics Letters, to be published.

[5] L.O. Heflinger, R.F. Wuerker and R.E. Brook, J. Appl. Phys. 37 (1966) 642 .

[6] R.L. Powell and K.A. Stetson, J. Opt. Soc. Am. 55 (1965) 1593.

17] B.P. Hildebrand and K.A. Haimes, J. Opt. Soc. Am. 57 (1967) 155.

[8] F.T.S. Yu, Introduction to diffraction, information processing and holography (MIT press, Cambridge, Mass. 1973) pp. 331-344.

[9] E.N. Leith, private communication. 